
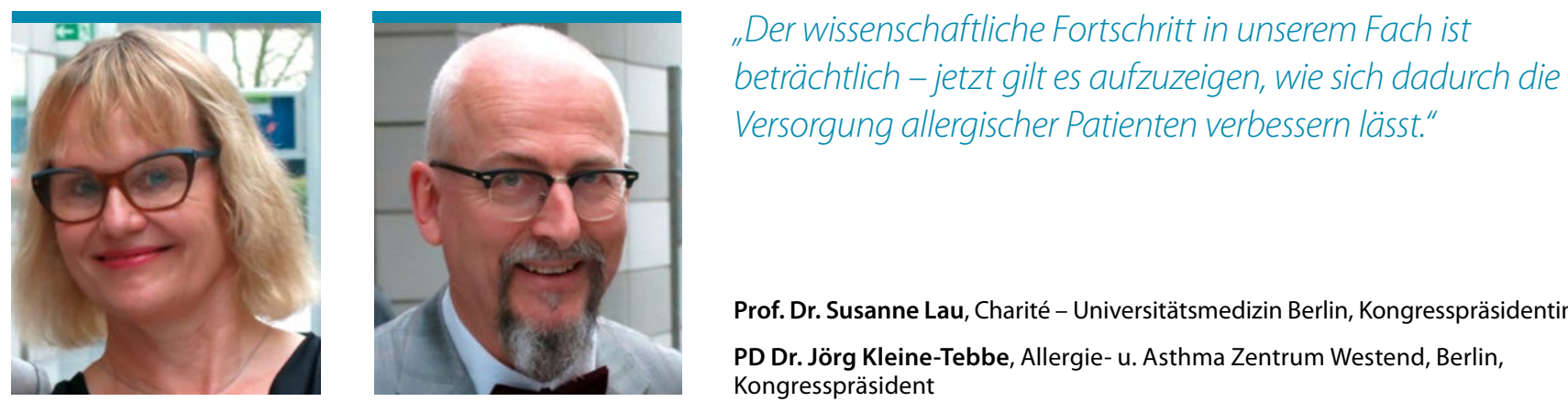

Prof. Dr. Susanne Lau, Charité - Universitätsmedizin Berlin, Kongresspräsidentin PD Dr. Jörg Kleine-Tebbe, Allergie- u. Asthma Zentrum Westend, Berlin, Kongresspräsident

\title{
Moderne Allergologie - Konzepte für die Zukunft
}

\section{L} iebe Kolleginnen und Kollegen, seien Sie herzlich eingeladen zum 11. Deutschen Allergiekongress (DAK), der vom 29. September bis 1. Oktober 2016 in Berlin stattfinden wird.

Das „Hauptstadt-Programm“ wird in großer Koalition entwickelt: Die Deutsche Gesellschaft für Allergologie und klinische Immunologie (DGAKI), der Ärzteverband Deutscher Allergologen (AeDA), und die Gesellschaft für Pädiatrische Allergologie und Umweltmedizin (GPA) ziehen dazu an einem Strang.

„Moderne Allergologie - Konzepte für die $\mathrm{Zu}$ kunft" - mit diesem Motto für den 11. DAK wollen wir bewusst eine dicke Lippe riskieren, ein paar alte Zöpfe abschneiden und neue Wege präsentieren. Die Allergologie muss raus aus der Schmollecke und ihre Bandagen abwerfen. Der wissenschaftliche Fortschritt in unserem Fach ist beträchtlich - jetzt

\section{Deutscher Allergiekongress auf einen Blick}

\section{Veranstalter}

_ Ärzteverband Deutscher Allergologen (AeDA)

_ Deutsche Gesellschaft für Allergologie und klinische Immunologie (DGAKI)

_ Gesellschaft für Pädiatrische Allergologie und Umweltmedizin (GPA)

\section{Kongresstermin und -Ort}

29. September bis 1. Oktober

Berlin Congress Center

\section{Kongresspräsidenten}

PD Dr. Jörg Kleine-Tebbe, Berlin

Prof. Dr. Susanne Lau, Berlin

\section{Anmeldung und Information}

Aktuelle Informationen sind im Internet unter www.allergiekongress.de zu finden. Die Anmeldung ist online möglich oder mit nebenstehendem Anmeldeformular per Fax. gilt es zu aufzuzeigen, wie sich dadurch die Versorgung allergischer Patienten verbessern lässt.

Getagt wird in Berlins Mitte am Alexanderplatz im Berlin Congress Center (bcc). „Wenn's voll ist, wird's gemütlich“" (alte Hauptstadtregel) und frische Kontakte gibt es umsonst dazu. Wir setzen auf bewährte Kongressformate: Plenarsitzungen, AllergoCompact, AllergoConnect, Industriesymposien, Pro-und-Con-Debatten in der AllergoArena und eine Reihe interdisziplinärer Workshops in Form der AllergoActives zu sämtlichen allergologischen Themen erwarten Sie in Berlin.

Auf der Grundlage neuer Forschungsergebnisse soll der klinische Umgang, die Vorbeugung, Diagnostik und Therapie allergischer Erkrankungen intensiv diskutiert werden. Sowohl Neueinsteiger als auch alte Hasen in der Allergologie sollen vom interaktiven und interdisziplinären Austausch beim 11. DAK profitieren. Gerade jüngere Mitglieder der Fachgesellschaften beziehungsweise Nicht-Mitglieder finden hier ideale Bedingungen zum wissenschaftlichen Networking oder Gelegenheit, in „Hands-on“-Veranstaltungen praktische Erfahrungen für den klinischen Alltag zu sammeln.

Am Donnerstagabend gibt es eine Berliner „Happy Hour" in der Industrieausstellung. Der Gesellschaftsabend am Freitag, noch in der Planung, soll Berlin von einer ganz anderen Seite zeigen - so wie Sie es vielleicht noch nie erlebt haben. Ihr Interesse ist hoffentlich geweckt. Jetzt runter von der Couch, die Termine (knapp vor dem Tag der Deutschen Einheit) blockieren und zügig anmelden, bevor es zu spät ist. Wir freuen uns, Sie in Berlin zu begrüßen!

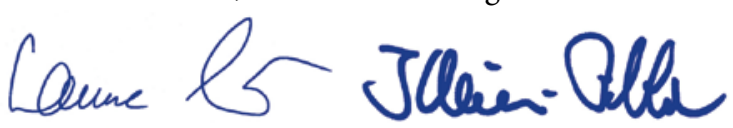

